Activités

16-1 | 2019

Comprendre le travail dans les "métiers adressés à autrui"

\title{
Les enseignants de la formation professionnelle : leur travail, leur réalité
}

Teachers in vocational training: the reality of their work

\section{Otilia Holgado}

\section{OpenEdition}

\section{Journals}

Édition électronique

URL : https://journals.openedition.org/activites/3995

DOI : 10.4000/activites.3995

ISSN : 1765-2723

Éditeur

ARPACT - Association Recherches et Pratiques sur les ACTivités

Référence électronique

Otilia Holgado, "Les enseignants de la formation professionnelle : leur travail, leur réalité », Activités

[En ligne], 16-1 | 2019, mis en ligne le 15 avril 2019, consulté le 21 septembre 2021. URL : http:// journals.openedition.org/activites/3995; DOI : https://doi.org/10.4000/activites.3995

Ce document a été généré automatiquement le 21 septembre 2021.

\section{cc) (i) $\Theta$}

Activités est mis à disposition selon les termes de la licence Creative Commons Attribution - Pas d'Utilisation Commerciale - Pas de Modification 4.0 International. 


\title{
Les enseignants de la formation professionnelle : leur travail, leur réalité
}

Teachers in vocational training: the reality of their work

\author{
Otilia Holgado
}

\section{NOTE DE L'ÉDITEUR}

Article soumis le 3 juin 2018, accepté le 14 février 2019

\section{Introduction}

1 Les recherches menées sur le plan international montrent que la formation professionnelle occupe une place importante dans le paysage éducatif, économique et social des pays, assurant la formation d'une main-d'œuvre qualifiée autrement difficile à trouver par les entreprises et les institutions. Toutefois, située à mi-chemin entre l'enseignement général et la formation en entreprise (Billett, 2011), la formation professionnelle a été longtemps et continue à être peu valorisée (Masdonati, Fournier, \& Pinault, 2015), considérée comme une « voie de garage » pour des élèves en difficulté scolaire dans le système d'enseignement général. Peu de moyens sont alloués pour permettre à la formation professionnelle de se réinventer ou de se renouveler afin de suivre l'incessante transformation des métiers (Allen, Harris, \& Butlin, 2003 ; Fournier, Bourassa, \& Gauthier, 2013 ; Santelmann, 2014). Ainsi, les changements observés se traduisent souvent par une complexification des prescriptions, normes et cadres qui régissent son fonctionnement (Achtenhagen \& Grubb, 2001). Du côté de la recherche, la formation professionnelle est un objet d'étude peu convoité, aussi bien par la recherche en sociologie (Jellab, 2005) qu'en sciences de l'éducation (Billett, 2011). Peu étudiée et 
documentée, l'activité des intervenants de la formation professionnelle est largement méconnue à la communauté scientifique (Billett, 2011 ; Jellab, 2005).

2 Au Québec, la recherche sur les enseignants de la formation professionnelle apporte un éclairage sur certains aspects, comme les conditions de formation et d'insertion des ceux-ci, mais peu sur leur travail au quotidien. Notons dans ce sens les travaux sur le choix de carrière et sur l'entrée dans le métier des enseignants débutants (Balleux, 2017; Deschenaux \& Roussel, 2008, 2010), sur l'accompagnement des ceux-ci (Coulombe, Zourhlal, \& Allaire, 2010), sur la formation à l'enseignement professionnel (Gagnon \& Coulombe, 2016) et sur certains aspects précis de l'enseignement, tels que l'évaluation (Roussel, 2014) ou encore la santé et la sécurité (Chatigny, Nadon-Vézina, Riel, Couture, \& Hastey, 2012).

3 En 2013, le constat partagé de méconnaissance des réalités vécues par les enseignants et les formateurs de la formation professionnelle a suscité un certain nombre d'interrogations. L'Institut Joseph Jacotot pour la formation professionnelle a alors mandaté plusieurs chercheurs français, suisses, belges et québécois pour explorer et caractériser leur travail et présenter publiquement leurs constats dans le cadre d'une conférence de consensus (2014). Notre participation à ces travaux collectifs a consisté à examiner et caractériser l'activité de travail des enseignants de la formation professionnelle scolaire dans le contexte québécois. Les aspects que nous avons examinés en priorité sont : les conditions d'embauche, les missions et les fonctions des enseignants, le public à former, les difficultés rencontrées sur le plan pédagogique, administratif et organisationnel. Le présent texte rend compte d'une partie des résultats obtenus dans le cadre de cette recherche.

\section{Un contexte historique et ses conséquences}

4 Contrairement à certains pays comme l'Allemagne, l'Australie ou la Suisse, où la formation professionnelle est organisée en partenariat entre les entreprises, les instances locales et les organisations scolaires, le Québec a fait le choix d'un modèle essentiellement scolaire pour « la production des compétences requises par le marché du travail» (Payeur, 1994, p. 2). Ainsi, que ce soit pour des jeunes ou pour des adultes en requalification, la responsabilité de la formation professionnelle est assurée exclusivement, depuis 1964, par le Ministère de l'Éducation. Déchargé de cette responsabilité, le Ministère du Travail détourne son regard de la formation scolaire. Quant aux sciences de l'éducation, Habboub, Lenoir et Tardif (2008) faisaient remarquer que cette discipline ne fait pas de la formation professionnelle une priorité en termes de recherche. Aujourd'hui la situation reste sensiblement la même au Québec. Dans ces conditions, la recherche avance grâce à l'investissement soutenu des universitaires intervenant dans la formation des enseignants de la formation professionnelle, en collaboration avec des acteurs de terrain. Toutefois, les travaux qui portent sur les enseignants de la formation professionnelle apportent peu d'éclairage sur leur travail, préférant s'intéresser principalement à leur formation (Tardif \& Deschenaux, 2014), aux conditions de leur entrée dans l'enseignement (Deschenaux \& Roussel, 2011; Deschenaux \& Tardif, 2016a), à leurs dynamiques identitaires (Grossmann, 2011), leur rapport au savoir (Beaucher, 2011), la transition de l'exercice d'un métier vers son enseignement (Balleux, 2017) ou l'accompagnement des débutants (Gagnon, 2013). 


\section{Au cœur de la formation professionnelle au Québec, les enseignants}

5 Au Québec, l'apprentissage professionnel se déroule principalement dans les centres de formation professionnelle, qui accueillent chaque année plus de 100000 jeunes et moins jeunes, en les conduisant vers l'obtention d'un diplôme d'études professionnelles. Pour dispenser la formation théorique et pratique dans chacun des plus de 150 programmes de formation organisée par compétences (MELS, 2005) et délivrée dans 195 centres de formation professionnelle (FCSQ, 2018) par des milliers d'enseignants recrutés parmi les travailleurs en emploi, sur un critère d'excellence dans leur métier d'origine. Ceux-ci débutent leur nouvelle carrière « au tournant de la quarantaine » (Balleux, 2017) engagés à l'essai et à la tâche, au gré des besoins plutôt fluctuants, du marché du travail. Enfin, ils n'ont pas, lors de l'embauche, de formation préalable en enseignement, mais s'engagent à poursuivre, sur leur temps libre, une formation appelée Baccalauréat en enseignement professionnel (BEP). Cette formation universitaire est équivalente à quatre années d'études à temps plein, mais, suivie à temps partiel et grâce à certaines reconnaissances des acquis, elle est complétée en 8 à 10 ans. Malgré ces spécificités, les enseignants de la formation professionnelle se voient attribuer le même statut professionnel que leurs collègues de l'enseignement secondaire général : l'appartenance à la profession enseignante. Cela pose au moins deux difficultés. Premièrement, le mépris ressenti de la part des collègues de l'enseignement général (Deschenaux \& Roussel, 2011) avec qui ils partagent la même catégorie socioprofessionnelle. Deuxièmement, le cadrage de leur activité et de leur formation par le "référentiel des douze compétences professionnelles en enseignement » dans lequel certains aspects sont peu représentatifs de la réalité de leur métier.

6 En plus des particularités professionnelles et académiques de ses enseignants (Balleux, 2017 ; Deschenaux \& Tardif, 2016a), la formation professionnelle se distingue également de l'enseignement général sur d'autres plans, dont les finalités et les spécificités du contenu en jeu (Alexandre, 2016) ou encore les caractéristiques des élèves (Gagnon, 2008), que nous allons également étudier en lien avec l'activité des enseignants.

7 Ces constats nous amènent à notre objectif de recherche, qui est de caractériser le travail des enseignants de la formation professionnelle. Les objectifs spécifiques de notre recherche sont de :1/ mettre en évidence les tâches, les responsabilités et la charge de travail ${ }^{1}$ telles que les enseignants se les représentent eux-mêmes, $2 /$ rendre compte des conditions de travail, des difficultés qu'ils rencontrent et des moyens qu'ils déploient pour y faire face au quotidien.

\section{Cadre conceptuel : de la tâche prescrite à la représentation du travail}

8 L'enseignement est une profession (CSÉ, 2004), mais est avant tout une activité de travail, c'est-à-dire une activité inscrite dans des rapports salariés (Mayen, 2012). Dans l'acception de la psychologie du travail et de l'ergonomie, l'activité représente ce que le professionnel met effectivement en œuvre pour réaliser les tâches qui lui sont assignées (Leplat, 2008). Au travail, l'activité est organisée autour de et en réponse à 
une série de tâches à réaliser (Leplat \& Hoc, 1983) et est soumise à des lois, normes, prescriptions (Leplat, 2008; Mayen, 2012) que la psychologie du travail invite à examiner en réalisant une analyse de la tâche préalablement à l'analyse de l'activité (Leplat \& Hoc, 1983). L'analyse de la tâche permet d'identifier les tâches spécifiques à un poste ou à une fonction donnée, de préciser la manière dont on s'attend à ce que ces tâches soient exécutées - et que l'on retrouve dans la littérature sous le terme de «prescription».

9 Identifier les tâches réelles des enseignants n'est pas sans difficulté. Saussez et Yvon (2014) notent la difficulté à saisir la globalité du travail des enseignants et l'écart reconnu entre la prescription et le déploiement effectif de l'activité au quotidien. Une partie des tâches à réaliser sont communiquées aux enseignants de manière explicite et conventionnée, dès leur embauche, à travers de documents officiels tels que des référentiels de métier, des contrats de travail ou des notes de service. En ce qui concerne les enseignants de la formation professionnelle, un rapport de recherche de l'IRSST (Chatigny et al., 2012) précise que la tâche hebdomadaire prévue pour les enseignants de la formation professionnelle est composée de 20 heures allouées à la tâche éducative et de 15 heures complémentaires, dédiées principalement à des rencontres de travail, à la préparation et à l'organisation du matériel et des locaux d'enseignement. Selon Leplat (1992), la tâche effective dépasse souvent la tâche prescrite. Dans le contexte actuel d'intensification du travail enseignant (Diarra, 2011 ; Tardif, 2012), on s'attend à ce qu'une fois entrés en fonction, les enseignants découvrent que leur travail ne se limite pas aux tâches conventionnées et que d'autres tâches, prescrites d'une manière plus ou moins formalisée, leur sont prescrites à différents niveaux : directement par le Ministère de l'Éducation, par la Commission scolaire qui est l'employeur direct des enseignants, par la direction du centre de formation professionnelle dans lequel ils exercent ou par le collectif d'enseignants du même département. Nous faisons ainsi le constat que l'analyse des documents officiels qui définissent les tâches de l'enseignant ne peut suffire pour déterminer ce que ceuxci ont et auront à faire.

10 Enfin, analyser le travail revient également à explorer l'ensemble de conditions de réalisation des tâches. Ces conditions perçues par le professionnel comme étant pertinentes pour la réalisation des tâches constituent la situation. Les situations avec lesquelles se confrontent les personnes au travail sont singulières, complexes, inédites et la manière de les appréhender est personnelle (Mayen, 2012). Une analyse du couple activité-situation est essentielle, permettant de comprendre non seulement ce que les professionnels font, mais également ce qu'ils ont à leur disposition pour réussir la réalisation de la tâche ou au contraire, quelles sont les difficultés qu'ils rencontrent et les obstacles qui empêchent le déroulement de l'activité tel que prévue ou souhaitée (Mayen, 2008). En effet, là où certains professionnels voient des contraintes, d'autres trouvent des opportunités, ce qui conditionne de manière différente la représentation que les personnes se font de leur propre travail et que Leplat (2000) appelle la tâche redéfinie.

\subsection{La tâche prescrite : le référentiel de métier?}

Dans le domaine de l'éducation, les tâches prescrites sont définies généralement par des référentiels de métier. Selon Cros et Raisky (2010): 
«Le référentiel est un outil de médiation normatif permettant aux activités humaines de s'y référer (de s'y rapporter) pour étudier un écart» (p. 106).

12 Au Québec, le travail de l'enseignant est défini en termes de compétences. L'un des documents officiels qui décrivent le travail enseignant est appelé « Le référentiel des douze compétences professionnelles en enseignement» (MEQ, 2001). Celui-ci est a priori un système de repères, d'abord pour les enseignants eux-mêmes, pour orienter leur activité au quotidien, mais aussi pour analyser leur activité et explorer l'écart entre la prescription et l'activité effective. Sans prendre en considération les spécificités des différents domaines d'enseignement (formation initiale, formation des adultes, formation professionnelle), ce référentiel présente un degré élevé de généralité. Les thématiques abordées par notre recherche trouvent une correspondance avec six des douze compétences du référentiel (Tableau 1).

Tableau 1. Correspondance entre des compétences du référentiel des douze compétences en enseignement et les thématiques de notre recherche.

Table 1: Relationship between competencies of the reference frame of the twelve teaching skills and the items of our research

\begin{tabular}{|r|l|l|}
\hline No. & Énoncé de la compétence du référentiel & Thématiques abordées \\
\hline 3 & $\begin{array}{l}\text { Concevoir des situations d'enseignement-apprentissage } \\
\text { pour les contenus à faire apprendre, et ce, en fonction } \\
\text { de l'effectif scolaire et en vue du développement des } \\
\text { compétences visées dans les programmes de formation. }\end{array}$ & $\begin{array}{l}\text { Objectifs } \\
\text { d'apprentissage visés } \\
\text { Préparation des cours }\end{array}$ \\
\hline 4 & $\begin{array}{l}\text { Piloter des situations d'enseignement-apprentissage } \\
\text { pour les contenus à faire apprendre, et ce, en fonction } \\
\text { des effectifs scolaires et en vue du développement des } \\
\text { compétences visées dans les programmes de formation. }\end{array}$ & Enseignement \\
\hline 6 & $\begin{array}{l}\text { Planifier, organiser et superviser le mode de } \\
\text { fonctionnement du groupe-classe en vue de favoriser }\end{array}$ & $\begin{array}{l}\text { Enseignement } \\
\text { Accompagnement des } \\
\text { l'apprentissage et la socialisation des élèves. }\end{array}$ \\
\hline 7 & $\begin{array}{l}\text { Adapter ses interventions aux besoins et aux } \\
\text { caractéristiques des élèves présentant des difficultés } \\
\text { d'apprentissage ou d'adaptation ou encore un handicap. }\end{array}$ & $\begin{array}{l}\text { Accompagnement des } \\
\text { élèves }\end{array}$ \\
\hline 9 & $\begin{array}{l}\text { Coopérer avec l'équipe-centre, les parents, les } \\
\text { différents partenaires sociaux et les élèves en vue de } \\
\text { l'atteinte des objectifs éducatifs de l'école. }\end{array}$ & $\begin{array}{l}\text { Travail en équipe } \\
\text { Relations avec la } \\
\text { hiérarchie }\end{array}$ \\
\hline 10 & $\begin{array}{l}\text { Travailler de concert avec les membres de l'équipe } \\
\text { pédagogique à l'exécution des tâches permettant le } \\
\text { développement et l'évaluation des compétences visées } \\
\text { dans les programmes de formation, et ce, en fonction } \\
\text { des élèves visés. }\end{array}$ & Travail en équipe \\
\hline
\end{tabular}

\subsection{Les représentations fonctionnelles des enseignants comme traces de leur activité}

13 Utilisée pour une large série de disciplines, la représentation des personnes ressort comme étant un objet de recherche pertinent lorsqu'on s'intéresse au métier tel que perçu par le professionnel lui-même. Jean Clénet (1998) considère que le concept de représentation permet d'aborder les aspects cognitifs et sociaux qui caractérisent la relation entre l'individu et le monde, la relation entre l'individu et l'action ainsi que la relation de l'individu avec lui-même. Quant à notre recherche, en utilisant l'entretien comme outil de recueil de données sur le travail, nous accédons à la fois aux 
représentations que les enseignants de la formation professionnelle ont construites à propos des tâches qui leur sont assignées, de l'activité mise en œuvre pour les réaliser, mais aussi des conditions de travail, c'est-à-dire des situations, consignes et contraintes qui conditionnent l'activité de réalisation de ces tâches au quotidien. Nous assistons ainsi à des manifestations de cette forme de représentation structurante (Ochanine, 1969) qui assure la planification et le guidage de l'action (Leplat, 1992 ; Vergnaud, 2007) et qui se retrouve dans la littérature sous plusieurs termes: représentation fonctionnelle (Leplat, 1992 ; Rogalski, 2013), représentation pour l'action (Weill-Fassina, Rabardel, \& Dubois, 1993) ou encore image opérative (Ochanine \& Zaltzman, 1969, 1973). On peut considérer que les représentations que les enseignants se sont construites de leur travail sont fonctionnelles dans la mesure où celles-ci leur permettent d'aménager leur quotidien de manière à faire face efficacement aux situations qui se présentent à eux. Plusieurs caractéristiques des représentations fonctionnelles ont été identifiées (Teiger, 1993 ; Vermersch \& Weill-Fassina, 1981). Nous en retenons leur caractère personnel, qui nous permet d'accéder aux « aménagements individuels spécifiques» (Valot, Grau, \& Amalberti, 1993, p. 272) que les enseignants mettent en place au quotidien. Les aménagements composent l'activité qui conduit à la "tâche effective " que Leplat (Leplat \& Hoc, 1983) invite à distinguer de la «tâche prévue ». Ces aménagements illustrent également l'écart par rapport à la prescription, ou au référentiel et que Cros et Raisky (2010) invitent à étudier. Nous retenons également la déformation fonctionnelle des représentations, qui représente l'accentuation des caractéristiques d'un objet (ou d'une situation) lorsqu'il revêt une importance toute particulière pour le sujet (Ochanine \& Koslov, 1971). Enfin, le caractère sélectif et le caractère lacunaire suscitent notre intérêt pour la pertinence attribuée aux informations qui ressortent à travers les représentations. Lorsqu'on analyse un métier à partir des représentations des travailleurs, la prise en considération de ces caractéristiques permet d'aller au-delà du travail prescrit et normé, pour s'intéresser aux aspects concrets et situés que les participants font ressortir. Toutefois, les données recueillies par entretien libre sont subjectives. Cellesci rendent compte de la tâche redéfinie (ou représentée) qui est en décalage avec la tâche réelle (Leplat, 1997). Il s'agit ici de l'une des limites de notre recherche.

\section{Aspects méthodologiques}

Les éléments conceptuels présentés, tirés de la psychologie du travail, de l'ergonomie et de la didactique professionnelle, invitent à une diversification des sources de données et des méthodes d'analyse permettant de saisir le travail de l'enseignant sur plusieurs angles. Nous avons choisi deux de ces angles. Premièrement, l'analyse du référentiel des compétences en enseignement nous a permis de connaitre la prescription générale $d u$ travail enseignant. Deuxièmement, l'analyse des représentations que les enseignants participants ont laissé voir à propos de leur travail, lors des entretiens, nous a apporté un point de vue personnel sur le travail effectué et sur la manière d'interpréter, de prendre en considération et de respecter la prescription.

15 Huit enseignants de la formation professionnelle ont participé à cette recherche qui s'inscrit dans une perspective compréhensive. Chacun d'entre eux enseigne dans un centre de formation professionnelle différent. Ils ont été recrutés sur une base 
volontaire. Comme nous ne nous intéressons pas aux enseignants débutants et que nous avons souhaité écarter autant que possible les préoccupations liées à l'entrée dans le métier, les critères d'âge (plus de 40 ans) et d'expérience en enseignement (plus de 5 ans d'expérience en enseignement) ont été introduits. Âgés de 40 à 60 ans, les participants ont entre 7 et 25 années d'expérience en enseignement et plus de 10 années d'expérience de travail dans le métier qu'ils enseignent. Les métiers spécialisés concernés par cette recherche sont : arpentage (E1), cuisine (E2), entretien paysager (E3), secrétariat (E4), transport par camion (E5 et E6), ainsi que la conduite d'engins de chantier (E7 et E8).

La méthode de recueil de données utilisée pour accéder aux représentations des enseignants a été l'entretien libre individuel. Il a été demandé à chaque enseignant participant de parler de son travail sous un angle personnel, sans se contraindre à des aspects prédéfinis. Les entretiens ont duré entre une et deux heures et ont été menés dans le centre de formation professionnelle de chaque participant. Les entretiens ont été enregistrés au moyen d'un enregistreur audionumérique, puis transcrits intégralement à des fins d'analyse. De manière tout à fait involontaire, la plupart des entretiens ont été entrecoupés par la réalisation, en notre présence, de certaines tâches professionnelles qui, vraisemblablement, ne pouvaient pas attendre : préparation des sorties sur le terrain, vérification du matériel, supervision d'élèves en atelier ou sur le terrain pédagogique, coordination entre collègues. Cette alternance d'activité concrète et de récit sur l'activité a permis aux enseignants de relier leurs témoignages à des « faits réels » que le chercheur peut suivre in situ.

17 Selon Pastré (2011, p. 133), « quand se déploie l'activité du sujet capable, cette activité se réfère aux situations, elle se réfère à autrui, elle se réfère au propre passé du sujet ». Pastré a fait ce constat lors des observations en situation de travail, mais nous remarquons que ceci est valable également lorsqu'il s'agit d'entretiens. En effet, lorsque l'entretien se déroule sur le lieu de travail, les conditions sont réunies pour que les professionnels parlent plus longuement et plus aisément de leur activité professionnelle, voire font la démonstration de leur activité en réalisant des tâches professionnelles en présence du chercheur. Toutefois, dans ces moments, leur récit ne porte pas uniquement sur des aspects qui concernent le moment présent. Ils expriment également leurs grandes préoccupations et les aspects saillants de leur travail.

Parmi les données recueillies, nous avons privilégié pour cet article celles qui ont permis de mettre en lumière la variété et les particularités des tâches évoquées par les enseignants participants de manière à montrer l'étendue de l'activité caractéristique du métier d'enseignant de la formation professionnelle au Québec. Après la transcription des enregistrements, les verbatims ont été découpés en épisodes, sur le critère de cohérence thématique. Le logiciel de traitement qualitatif NVivo a été utilisé pour attribuer les épisodes aux catégories thématiques retenues, soit : l'enseignement, les objectifs d'apprentissage, la préparation des cours, le travail en équipe, l'accompagnement des élèves, les autres tâches. 


\section{Analyse des résultats : activité des enseignants de la formation professionnelle}

\subsection{L'activité d'enseignement, une constante à configuration variable}

19 Au Québec, un contrat annuel à temps plein engage les enseignants à 1280 heures par an de travail avec présence dans le centre de formation, dont 720 heures d'enseignement, sauf pour le programme de transport par camion, où les enseignants assurent 864 heures d'enseignement effectif par an. Cela signifie que l'enseignement effectif occupe $56 \%$ de leur temps de travail ( $72 \%$ en transport par camion). Les enseignants passent donc plus de la moitié de leur temps de travail en compagnie des élèves : 3 ou 4 jours par semaine, 6 à 7 heures par jour. Ce programme convient aux enseignants, le " volume d'heures » passées en compagnie des élèves n'est pas pour eux une contrainte, bien le contraire. C'est avec beaucoup de sérénité que cet enseignant qui a choisi de travailler « du soir » décrit sa journée :

«On commence à 2 heures l'après-midi. On rentre du cours [qui a eu lieu dans le camion, sur la route, en roulant] vers 9 heures et demie. On dételle, on remise puis vers 10 heures [du soir] on s'en va » (E5).

Le même état d'esprit caractérise cet autre enseignant qui, lui, raisonne en nombre d'heures consécutives à dédier à un module d'enseignement précis : «J'ai 35 jours, 240 heures, pour les amener à l'évaluation " (E8), ce qui correspond à une moyenne de 6,8 heures d'enseignement par jour, durant 35 jours ouvrables consécutifs. Tous les enseignants témoignent d'un programme serré et du fait que celui-ci ne laisse pas place à des imprévus. Pourtant, des imprévus interviennent régulièrement et souvent les enseignants n'ont pas d'autre choix que de les prendre sur leur temps personnel. Il s'agit alors d'une contrainte :

« On a un horaire à temps plein. Quand un élève échoue un examen, faut qu'il puisse le reprendre. Mais il n'y a pas de temps pour le faire dans l'horaire. Alors il faut que

l'enseignant soit disponible » (E4).

Nous constatons ici que l'activité d'enseignement dépasse la tâche prescrite, aussi bien en termes de temps que de tâches (reprises d'examens). Tous les enseignants témoignent d'un programme chargé, organisé selon des contraintes organisationnelles qui ne sont pas toujours adaptées à l'ampleur des apprentissages prévus. De plus, les tâches à réaliser ont des durées variables, alors que les séquences de formation ont une durée fixe. Cela pose parfois des difficultés d'ordre pédagogique : nécessité de morceler les tâches à réaliser, passer rapidement sur certaines opérations, notamment lors des phases de préparation et de finalisation. Cela amène les enseignants à réaliser, quotidiennement, avant l'arrivée des élèves et après leur départ, un certain nombre de tâches indirectement liées à l'enseignement et qui ne peuvent pas être faites avec les élèves par manque de temps. C'est souvent le cas de l'entretien, la vérification et l'entreposage des équipements et du matériel. En formation professionnelle, un entretien régulier et méticuleux qui ne peut pas être négligé, la santé et la sécurité des élèves étant en jeu. Il ne s'agit pas de ranger le matériel de laboratoire sur des étagères, mais de vérifier et d'amener les camions au garage, d'affuter les couteaux utilisés, de vérifier l'équipement de sécurité de chaque élève, de finir d'emballer et réfrigérer les plats cuisinés préparés. Cette tâche prend des proportions considérables, supérieures au temps comptabilisé dans la tâche professionnelle des enseignants. Pour mieux 
s'organiser dans ce contexte, les enseignants apprécient avoir un programme d'enseignement régulier. Pour cela, lorsque c'est possible, les centres de formation professionnelle pratiquent une organisation de l'enseignement pas modules. Chaque module est pris en charge par un enseignant sur toute sa durée, ce qui permet l'installation d'une activité routinière que les enseignants évoquent comme une des clés de la réussite des élèves, mais aussi comme une qualité de leurs conditions de travail et qu'ils souhaiteraient conserver :

«Des fois j'enseigne 3 heures, des fois 6 heures, des fois une journée. Deux différents groupes, deux matières différentes, la même journée. Je n'aime pas ça, c'est bon pour personne. » (E2)

Les enseignants participants disent assurer leurs tâches d'enseignement avec rigueur. Ils se représentent l'apprentissage professionnel aussi comme une préparation aux conditions de travail et déplorent le fait que les élèves ne sont pas toujours dans ce même état d'esprit, comme le témoigne l'activité de cet enseignant en cuisine : «je regarde... Il n'arrivera jamais. Bouge! Le chef ne te gardera pas. » (E2) ou alors, le même enseignant: «j'aime le travail bien fait (...), mais je ne peux pas demander aux élèves d'être comme moi.»

Ce constat corrobore les résultats d'autres recherches. Comme le constatent Deschenaux et Roussel (2011) :

«La rigueur représente une qualité qui marque fréquemment les propos des enseignants. Certains semblent même s'enorgueillir de leur rigueur, voire de leur sévérité. En fait, la plupart du temps, lorsqu'il est question de rigueur, cela équivaut davantage à la conformité liée aux exigences de la pratique du métier qu'ils enseignent. » (p. 23)

En revanche, selon les mêmes auteurs, les enseignants «insistent rarement sur la rigueur dans les contenus ou la correction des examens.» (Ibid., p. 23) Nos données nous amènent effectivement au même constat, comme le témoigne cet enseignant en transport par camion : " y en a, c'est long de développer la compétence, mais ils sont bien... je les trouve très compétents dans le milieu, mais ça va être plus long de développer la maîtrise du véhicule». Les examens sont parfois imposés aux enseignants. D'autres fois, les enseignants participent à la conception des épreuves et des grilles d'évaluation. Quelles que soient les modalités d'évaluation mises en place, les enseignants se disent insatisfaits et soulignent les limites de l'évaluation en formation professionnelle. Selon eux, la réussite aux examens ne correspond et n'atteste pas toujours et pas suffisamment des acquis d'ordre professionnel directement exploitables sur le marché du travail. En même temps, nous notons une volonté de la part des enseignants de respecter les prescriptions et pratiquer une activité évaluative de type scolaire. Certains y arrivent: " je me limite à ce que l'évaluation me demande » (E1). Pour d'autres, cette volonté entre en tension avec la manière dont ils se représentent l'évaluation des compétences professionnelles et des apprentissages qui préparent effectivement les élèves pour le marché du travail. Toutefois, ces tensions se réduisent lorsque les enseignants arrivent à interpréter les consignes de manière à faire évoluer leurs représentations de l'évaluation scolaire vers une formule mixte, scolaire et professionnelle, qui devient alors acceptable (Holgado \& Nizet, 2016). 


\subsection{Objectifs d'apprentissage visés}

24 formation spécifique à chaque métier, défini au niveau provincial. Étant données les multiples possibilités d'emploi liées à un même métier, les objectifs d'apprentissage sont orientés par les enseignants vers le développement de la polyvalence, comme en témoigne cet enseignant en conduite d'engins de chantier: «ils vont les apprendre toutes [les machines]» (E7). D'autres objectifs visant le développement de compétences de nature plus administrative sont également au programme: curriculum vitae, recherche documentaire sur les entreprises, démarchage d'un stage. Les enseignants évoquent ces objectifs comme étant «des consignes à suivre ». Ils précisent que ces objectifs « visent à assurer un premier niveau d'autonomie » (E3) et ne s'attardent pas sur ces aspects lors de leur témoignage. Mais que signifie cette visée d'un début d'autonomie? Du fait que "l'école n'est pas l'industrie » (E2) et que le niveau de compétence recherché durant la formation est difficile à déterminer et à formaliser dans des prescriptions, les enseignants ont un espace de liberté quant au niveau de compétence à atteindre par les élèves. Quand ils utilisent cet espace de liberté pour définir le niveau de développement des compétences à atteindre, ce n'est pas seulement la maitrise du geste qui est recherchée, ni l'endurance ou l'efficience, mais la conceptualisation des actes et de leurs effets :

«Quand il est capable de me dire : regarde là, j'ai mal fait pour telle raison, moi dans ma tête : il a compris. C'est juste une question d'adresse qu'il va aller chercher avec de la pratique » (E8).

Les enseignants font également jouer leur créativité sur les modalités permettant d'atteindre les objectifs imposés : «j'ai carte blanche dans mes cours, c'est-à-dire les objectifs sont ça, mais tu choisis la façon de les atteindre " (E6), ainsi que sur les attitudes professionnelles qu'ils se font un point d'honneur à développer chez les élèves, comme le respect : « le respect des autres (...) et le respect de soi, marcher la tête haute, être fier de qui on est » (E1), le plaisir : « rendre ça plus agréable » (E3), la curiosité : « aller plus loin, explorer ce qui nous rapprocherait encore plus du milieu du travail » (E4). Il y a également la sensibilisation au respect des normes de sécurité, que les enseignants se représentent à la fois comme une nécessité «quand qu'ils vont en industrie, y sont responsables de leur propre sécurité » (E5) et comme une limite du système scolaire: "à l'école, on essaie de toujours les mettre dans des situations sécuritaires pour ne pas qu'il arrive d'accidents » (E5).

Enfin, les enseignants sont formels : se préparer pour le monde du travail ne revient pas à réussir les examens, pas seulement. Pour cette raison, la réussite des élèves aux examens n'est pas pour eux un objectif en soi :

«Moi, c'est sur la compétence... au-delà de l'examen. Parce qu'on peut avoir la compétence puis échouer un examen (...) et l'inverse, on peut réussir quelque chose qu'on nous demande sans comprendre le pourquoi »(E1).

Certains enseignants sont plus stricts sur ce point, non sans regret :

«Les préparer pour un examen, c'est le but dans tous les cours. Enfin... c'est dommage... c'est la queue qui branle le chien » (E5).

Du point de vue des objectifs d'apprentissage visés, les enseignants composent avec la prescription et le degré de liberté pédagogique et didactique dont ils disposent pour préparer les élèves à intégrer et à affronter le monde du travail. Tout en restant respectueux du programme scolaire prescrit et à l'apprentissage du métier, ils guident 
également les élèves vers le développement d'une compétence sociale recherchée par les employeurs et qui est basée sur des attitudes face au travail : créativité, curiosité, respect, plaisir du travail bien fait. Dans cet espace d'apprentissage qu'ils construisent, les enseignants trouvent une marque de reconnaissance de leur propre compétence, à la fois comme professionnels (maîtres du contenu) et comme enseignants (maîtres des modalités).

\subsection{Activité de conception et de préparation des cours}

Une recherche réalisée en Suisse, pour le canton de Genève (Ducrey, Hrizi, \& Issaieva Moubarak-Nahra, 2010) montre que les enseignants de la formation professionnelle genevoise passent en moyenne $40 \%$ de leur temps de travail en classe avec les élèves et autres $40 \%$ pour la préparation et la planification des cours, la construction et la correction des évaluations. Les enseignants genevois se déclarent satisfaits de bénéficier d'une heure de préparation pour une heure d'enseignement. Étant donné que les enseignants québécois de la formation professionnelle passent plus de $50 \%$ de leur temps de travail en présence des élèves, ils ne peuvent pas consacrer tout autant de temps à la préparation des cours étant donné les multiples autres tâches, ce qui entraine une surcharge de travail. Par conséquent, ils prennent sur leur temps personnel la préparation des cours, notamment en début de carrière. Cette situation a des implications au niveau familial :

« Ça faisait des grosses semaines, la préparation et la correction tout ça, ça se faisait tout à la maison. (...) moi, je l'ai bien vécu, on avait pris la décision que Marie restait à la maison, donc je n'avais pas cette inquiétude-là au niveau des enfants » (E1).

Pour d'autres enseignants en revanche, le partage entre la vie professionnelle en début de carrière et la vie familiale était plus délicat :

" Je couchais ma dernière, je sortais de sa chambre il était 8 heures et demie, puis je travaillais jusqu'à 11 heures et demie, minuit. Pendant un mois et demi. C'est trop de travail. Avec deux petits enfants... moi, je n'y arrive pas » (E2).

L'emploi des verbes au passé nous indique que les enseignants font référence à des expériences passées, donc à un état temporaire, qui caractérise principalement l'entrée dans le métier. Toutefois, si la surcharge de travail liée à la préparation des cours s'allège avec le temps, celle-ci ne disparait pas, mais se gère différemment :

«Aujourd'hui, au niveau de la préparation c'est plus facile... présentement, c'est sûr que je suis en fin de carrière... c'est bien, bien rempli, mais je fais tout à l'école » (E1).

Malgré ces désagréments, les enseignants ne se représentent pas cette partie de la surcharge de travail comme une contrainte, mais plutôt comme une condition nécessaire à la réussite de leur entrée, voire de leur poursuite dans la profession.

En formation professionnelle, la préparation des cours ne se limite pas à la recherche documentaire et le montage des séquences pédagogiques. Comme l'activité se déroule le plus souvent à l'extérieur et parfois loin des bâtiments de l'école, l'enseignant est contraint à une planification rigoureuse et anticipative, car les possibilités d'ajustement peuvent rapidement trouver leurs limites. En entretien paysager par exemple, les enseignants doivent trouver des terrains pour la pratique. Cette tâche alourdit considérablement leur temps de travail réel, étant extrêmement chronophage et possible uniquement grâce aux relations que les enseignants développent avec les propriétaires des terrains à proximité de l'école. Cette fois, les relations qu'ils ont gardées avec le milieu du travail et les entrepreneurs de la région ne leur sont d'aucune 
utilité. Plus que cela: régulièrement, ils prennent le temps de rencontrer les entrepreneurs pour les rassurer quant à une possible concurrence que le centre de formation pourrait leur faire auprès des clients de la région.

Lorsqu'il reste modéré, le recouvrement des deux dimensions, professionnelle et familiale, n'est pas vécu comme une contrainte, mais comme une réalité du métier. En effet, les enseignants parlent de leur vie professionnelle en enseignement non pas comme d'un travail, mais comme d'un mode de vie. Ils aiment l'enseignement, ils aiment le métier qu'ils enseignent et ils aiment le travail bien fait et ils ne comptent pas les heures nécessaires pour transmettre aux élèves la passion de ce métier.

\subsection{Travail en équipe}

31 Nous pouvons ainsi noter, tout comme Roussel (2014) le constate également, que d'une manière générale, la surcharge de travail amène les enseignants à travailler en équipe, à partager certaines tâches, à s'entraider. Toutefois, si Roussel fait notamment référence aux tâches pédagogiques (poser des gestes pour enseigner, maintenir un climat de classe approprié, ou évaluer) et constate que les possibilités d'aide entre enseignants sont assez limitées, nous constatons une réelle entraide sur le plan didactique qui fait preuve d'efficacité en raison de l'expertise professionnelle partagée par les enseignants: "selon nos spécialisations, on se répartit les modules » (enseignant en arpentage) ou encore :

«On a tous nos spécialités. Comme moi : j'étais spécialiste de flatbed, les remorques à plateau. Bien, quand viennent les ateliers de remorques à plateau, c'est moi qui va être le chef. Je vais superviser, je vais gérer, je vais m'occuper de tout ça » (E5).

Il y a également la coordination nécessaire entre les enseignants qui ont la charge simultanée des modules complémentaires: en conduite d'engins de chantier, après l'équipe qui fait des excavations passe l'équipe qui comble et qui nivèle. L'activité des uns conditionne en permanence l'activité des autres. La nécessité de pouvoir compter sur ses collègues pour réaliser ses tâches d'enseignement soude les équipes d'enseignants. Dans certains centres de formation, l'entraide fonctionne également pour la préparation des cours et permet aussi aux enseignants débutants une entrée dans le métier plus sereine, facilitée par la mise en commun des documents produits individuellement par les enseignants, comme le témoigne cet enseignant en transport par camion :

«Tout le monde partage ses choses. Le nouvel enseignant, il arrive, il a plein de documents... On est vraiment privilégié. Fait que... c'est correct qu'on travaille plus. » (E6)

\subsection{Activité d'accompagnement des élèves}

Selon les données ministérielles (MELS, 2014), l'âge des élèves inscrits dans un cursus de formation professionnelle scolaire et qui partagent la même classe est très variable : moins de $20 \%$ des élèves ont moins de 20 ans, plus de $30 \%$ des élèves ont plus de 30 ans. Ces élèves ont également des niveaux scolaires, des connaissances et des aspirations différentes, ce qui rend particulièrement difficiles les conditions de travail des enseignants (MEQ, 2001). Ledent et Schevers (2016) précisent que les enseignants montrent une attention toute particulière à la relation entretenue avec les élèves, qu'ils considèrent comme étant leurs élèves et avec qui ils développent une relation 
indissociable de leur fonction. C'est ce que nous avons également constaté. Les enseignants qui ont participé à notre recherche se représentent tous l'accompagnement extra-scolaire des élèves comme partie intégrante de leurs tâches de travail : « c'est mon métier aussi, là » (E1). En effet, passer des journées entières avec un groupe d'élèves créé nécessairement des proximités, sinon une intimité propice à des confidences : « ils me voient plus comme un grand-frère, ou un jeune père, fait que j'ai eu des confessions des fois » (E7).

Pour offrir cet accompagnement aux élèves, les enseignants se rendent disponibles en dehors du temps scolaire : " souvent des élèves restent après la classe, où viennent de bonne heure avant le cours, je suis tout le temps disponible » (E8). Ils considèrent ces échanges avec les élèves comme indissociables de l'enseignement. Premièrement, parce qu'ils peuvent connaitre les aspirations des élèves et les aider à les poursuivre : « il y avait un rêve en avant de ça [la formation suivie]» (E3). Cela permet également de les sensibiliser aux exigences du métier et de les orienter sur des choix qui leur correspondant : «il faut les ramener à la réalité du métier aussi » (E5). En effet, qu'il s'agisse de la cuisine, de l'entretien paysager ou du transport par camion, les enseignants tentent de préparer les élèves à la pénibilité des métiers spécialisés et au fait que ceux-ci sont « un job non traditionnel ». Ensuite, il s'agit d'entretenir un réseau professionnel en gardant contact avec les élèves après l'obtention de leur diplôme et leur arrivée sur le marché du travail. Les anciens élèves représentent un bassin d'emploi intéressant pour les élèves actuels : « un élève qui demande d'aller travailler à tel endroit, bien, on le met en contact avec un ancien élève » (E2). Enfin, parce que les confidences sont une modalité efficace de connaitre les problèmes extra-scolaires qui pourraient nuire à la sécurité de l'élève en question et même de tout le groupe : «ils ont besoin d'une oreille. Je les écoute puis je me rends compte qu'ils ne sont pas aptes à conduire »(E5).

\subsection{Activité de gestion, autres tâches et rapport à la hiérarchie}

Parmi les tâches considérées par les enseignants de la formation professionnelle comme des contraintes, il y a les tâches administratives. Ces tâches ne sont pas propres à l'activité des enseignants de la formation professionnelle. Dans certains centres de formation, un enseignant est désigné pour assurer la fonction de direction de département, ce qui entraine des tâches spécifiques d'organisation, commande d'équipement et de matériel, inventaire, gestion des budgets, participation à des comités, mais aussi un dégagement d'un certain nombre d'heures de cours, arrangement qui satisfait les enseignants concernés. Dans d'autres centres en revanche, les enseignants se partagent la responsabilité des modules du programme enseigné. Cette responsabilité implique des tâches administratives, logistiques et d'organisation: horaires, gestion des locaux et des équipements, commandes de matière première, etc., mais ce sont les tâches administratives que les enseignants redoutent le plus : «La pire contrainte, c'est la contrainte administrative» (E3). Pour certaines tâches, ils ne sont absolument pas préparés, comme pour faire les horaires du département. Leur activité est aléatoire, sans règles ni méthode, quoiqu'ils tentent de réaliser cette tâche du mieux qu'ils peuvent. Considérée comme aliénante, cette tâche de fait pas de sens pour eux: "jouer avec les cases horaires, en fonction des disponibilités des locaux, les vacances des professeurs... C'est aliénant »(E2). Les enseignants sont également responsables de l'entretien des locaux, du terrain 
pédagogique et du matériel. Lorsque c'est possible, ils effectuent eux-mêmes certaines réparations. Cette partie de la tâche, ils se la représentent comme étant nécessaire, quoique peu valorisante et assez fatigante. En revanche, ils déplorent les réunions qu'ils qualifient d'accablantes, notamment pour l'investissement et la préparation que celles-ci nécessitent : «c'est beaucoup de préparation, plus que les gens pensent. (...) Je n'enseigne plus beaucoup, je fais juste le travail de paperasse puis des réunions » (E2). Une équipe soudée ne voit pas la nécessité de ces réunions, parce que les échanges ont lieu en permanence, dans les couloirs, le bureau commun ou pendant les repas, que les enseignants prennent en commun.

D'autres tâches que les enseignants de la formation professionnelle ont à réaliser au quotidien semblent les distinguer, du moins en partie, des enseignants de l'enseignement général. Au quotidien, leur activité dépend de l'activité de nombreux autres acteurs : certes, leurs supérieurs hiérarchiques, leurs élèves, leurs collègues, mais aussi les professionnels de l'industrie, les fournisseurs, les concessionnaires. De plus, comme nous l'avons déjà montré au début de ce texte, le volume de travail nécessaire, notamment en début de carrière, rend la conciliation travail-famille difficile. Ainsi, les enseignants de la formation professionnelle se trouvent au centre d'un système complexe qui les transforme malgré eux. Ils inscrivent très fortement leur activité dans un cadre, celui de l'institution qui les emploie, mais dont ils s'approprient difficilement la culture. Et inversement, ils ont des difficultés à se faire entendre sur leur culture et leurs besoins : « lui [le directeur] nous écoute, il fait des efforts, mais ne comprend pas tout, il ne connait pas le métier » (E5). Habitués au régime alerte de la production, ces anciens travailleurs ont du mal à accepter que les démarches administratives puissent ralentir le déroulement de leur activité prévue. Ainsi, ils décrivent une relation plutôt positive ou neutre avec la direction du centre de formation, mais froide et distante avec les décideurs de la commission scolaire, qui, "d'en haut de leur tour de cristal» (E6), ne semblent pas comprendre le fonctionnement et les besoins de la formation professionnelle. Il y a ensuite les contraintes syndicales qui sont évoquées et selon lesquelles le critère d'ancienneté prime sur la spécialisation ou le degré d'expertise dans l'attribution des cours. Les enseignants débutants peuvent ainsi se retrouver à prendre en charge l'enseignement de certains modules pour lesquels ils ne se considèrent pas spécialistes, alors que leur compétence dans d'autres domaines est sous-exploitée.

Mais les principales contraintes, celles qui les distinguent le plus de leurs collègues de l'enseignement général, sont liées aux conditions inhérentes à l'apprentissage d'un métier dans des situations proches de celles qui caractérisent de travail réel et notamment lorsque l'activité se déroule à l'extérieur : les conditions météorologiques, les contraintes spatiales, temporelles et budgétaires. Dans certains métiers, l'enseignement se fait majoritairement à l'extérieur. L'activité est donc soumise aux aléas météorologiques qui affectent le fonctionnement de certains équipements : « une niveleuse, on ne peut pas aller dans la boue avec ça, il n'y a rien à faire.» (E6), mais aussi l'alternance des saisons, car les plantations et les terrains ne sont pas accessibles durant l'hiver et les enseignants doivent s'organiser pour que les activités estivales, automnales et printanières se produisent avant les premières neiges, pour que les élèves aient le temps d'apprendre les techniques nécessaires avant de pouvoir continuer la formation par des situations simulées : "faut s'arranger avec la nature. » (E3) 
38 En ce qui concerne l'alternance des groupes de jour et de soir, les enseignants expliquent devoir également adapter la supervision, les élèves étant trop distancés des lumières installées sur le terrain (ou sur la route, pour le transport par camion) pour pouvoir observer leurs manœuvres. Il y a ensuite la contrainte spatiale. Les terrains pédagogiques sont grands, les chantiers sont éloignés les uns des autres, ce qui limite la supervision et transforme l'activité d'enseignement prévue sous la forme d'un cours collectif en enseignement individualisé : «le champ c'est pas mal grand. Si c'est trop éparpillé, les élèves sont laissés à eux-mêmes et on ne peut pas enseigner à tout le monde en même temps » (E6). Contrairement à une salle de classe dans laquelle tous les élèves sont regroupés autour de l'enseignant, l'enseignement en atelier ou sur le terrain ne permet pas aux enseignants de fournir si aisément des explications supplémentaires aux élèves qui en auraient besoin. Comme l'explique un enseignant, il lui est impossible de passer un temps prolongé avec un élève en laissant les autres sans surveillance. Évidemment, dans cette situation, le fait d'avoir à enseigner à des élèves ayant des expériences différentes s'avère contraignant pour l'enseignant et limite son activité pour une raison de sécurité. En plus de cela, la décision de la direction d'agrandir les groupes compliquera encore plus l'activité d'enseignement.

\section{Conclusion}

Par cet article, nous avons souhaité présenter un portrait des tâches et des conditions de travail des enseignants de la formation professionnelle au Québec. Campbell (2017), qui s'est intéressé aux intentions de développement professionnel des enseignants de tous niveaux et secteurs scolaires confondus à travers les différentes provinces du Canada, a noté la présence d'une grande diversité de contextes, expériences et besoins au regard de la formation et du travail des enseignants, concluant à la nécessité de mener des recherches spécifiques qui prennent en compte les particularités locales.

Les résultats issus de cette recherche ont, évidemment, une portée locale. À travers le Québec, 170 centres de formation professionnelle embauchent actuellement plus de 10000 enseignants et accueillent chaque année plus de 100000 élèves (Deschenaux \& Tardif, 2016b). Il serait donc illusoire de penser pouvoir dresser un portrait dans lequel tous ces enseignants pourraient se reconnaitre. Toutefois, nous avons focalisé sur les dimensions du travail qui sont ressorties chez tous (ou la plupart) des enseignants participants. Nous avons ainsi pu constater que malgré les spécificités du travail dans chaque centre de formation et dans chacun des six programmes de formation pris en considération, certains aspects faisaient l'objet de représentations partagées de la part des enseignants. Nous avons tenté de faire ressortir les aspects saillants pour chacune des thématiques choisies. Ainsi, si les six thématiques retenues ne pourront circonscrire à elles seules le travail de ces enseignants, celles-ci nous ont permis d'esquisser quelques traits de celui-ci, pour apporter un éclairage - partiel et exploratoire - de la réalité du travail de ces enseignants.

41 Sur le plan de la méthode, nous avons souhaité donner autant que possible la parole aux enseignants. Nous nous sommes pour cela risquées à l'exercice d'intégrer des extraits d'énoncés des témoignages à même la présentation et la discussion des résultats, traitées simultanément. Ne pouvant pas reprendre les propos de tous au regard de chacune des thématiques abordées, nous avons essayé d'illustrer les propos communs par l'extrait de la transcription que nous avons considéré comme le plus 
caractéristique. Pour cela, nous n'avons pas porté attention à la parité des propos de chaque enseignant.

Sur le plan théorique, les représentations que les enseignants nous ont livrées de leur métier nous ont permis de rentrer assez finement dans leur activité quotidienne et d'en sortir des tâches caractéristiques ainsi que des formes d'activité, plus ou moins efficaces, par lesquelles les enseignants tentent d'accomplir ces tâches. Les représentations que les professionnels se sont construites de leur travail et qui leur permettent d'agir au quotidien s'appuient généralement sur un ensemble indissociable de tâches, dont certaines se retrouvent dans la prescription et d'autres non. Tel que présenté par Davezies (2012), dans le contexte actuel d'intensification du travail, les professionnels n'arrivent pas à accomplir la totalité du travail dans le temps attribué, les tâches prescrites et les tâches non prescrites, mais essentielles au bon fonctionnement de l'organisation étant bien plus nombreuses et plus prenantes que prévu.

43 L'activité quotidienne des enseignants de la formation professionnelle se structure autour de trois axes :

- Activité centrée sur l'enseignement, donc à visée didactique et pédagogique ;

- Activité centrée sur les élèves et les conditions de leur réussite, donc à visée professionnalisante et socio-éducative ;

- Activité centrée sur les tâches bureautiques et de gestion à accomplir, donc à visée administrative.

En regardant le référentiel des compétences en enseignement, nous pouvons constater que plusieurs tâches qui occupent une place importante dans le quotidien des enseignants de la formation professionnelle ne s'y retrouvent pas. Il s'agit d'une partie des tâches qui ne portent pas directement sur l'enseignement, comme celles que nous avons regroupées dans la catégorie " activité de gestion, autres tâches et rapport à la hiérarchie » et qui ne sont ni reconnues, ni valorisées, mais occupent une bonne partie du temps de travail des enseignants. C'est l'une des raisons pour lesquelles l'activité réelle des enseignants de la formation professionnelle dépasse leur temps de travail réglementaire. En réalité, seule l'activité d'enseignement est invariable, principalement parce que les cours et les activités pédagogiques ont une durée déterminée et se déroulent nécessairement pendant des plages horaires prédéterminées. En dehors de cela, il y a le travail invisible, non reconnu et non valorisé, mais nécessaire au bon fonctionnement du centre de formation et à l'apprentissage des apprenants.

Malgré ces contraintes, les enseignants ne semblent vouloir négliger aucune des tâches dont ils ont la charge, même celles qu'ils apprécient moins ou celles pour lesquelles ils se sentent moins compétents ou qu'ils prennent beaucoup de temps à accomplir. C'est ainsi que nous avons pu observer chez tous les enseignants participants à cette recherche que la limite entre la vie professionnelle et la vie familiale est floue.

$\mathrm{Au}$ Québec, lorsqu'ils débutent leur carrière, les enseignants de la formation professionnelle sont dans l'obligation de suivre, en parallèle à l'enseignement et à temps partiel, une formation universitaire orientée, en grande partie, vers la pédagogie. Si une formation en gestion administrative pourrait également aider ces enseignants à surmonter une partie des difficultés rencontrées au travail et leur permettre de limiter le dépassement du temps de travail réglementaire, s'est par une meilleure visibilité au sein de la profession et une compréhension des spécificités de 
leur métier par leur hiérarchie que les conditions de travail des enseignants de la formation professionnelle pourraient être améliorées.

\section{BIBLIOGRAPHIE}

Achtenhagen, F., \& Grubb, W. (2001). Vocational and occupational education: pedagogical complexity, institutional diversity. In V. Richardson (Ed.), Handbook of Research on Teaching. New York : Mc Millan.

Alexandre, M. (2016). Le processus didactique en enseignement professionnel : une étude de cas en Techniques d'éducation à l'enfance. In C. Gagnon \& S. Coulombe, Enjeux et défis de la formation à l'enseignement professionnel. Québec : Presses de l’Université du Québec.

Allen, M., Harris, S., \& Butlin, G. (2003). Finding Their Way: A Profile of Young Canadian Graduates. Statistics Canada Catalogue. Ottawa, ON : Statistics Canada.

Balleux, A. (2017). Du métier exercé au métier enseigné : transition, ruptures et continuités. Éducation permanente, 212(3), 27-35.

Beaucher, C. (2011). Le rapport au savoir, quelques fondements et pistes. La Webéducation, http:// www.webeducation.ca/

Billett, S. (2011). Vocational Education: Purposes, traditions and prospects. Springer : Dordrecht, The Netherlands.

Campbell, C. (2017). Developing Teachers' Professional Learning: Canadian Evidence and Experiences in a World of Educational Improvement. Revue canadienne de l'éducation, 40(2). http:// journals.sfu.ca/cje/index.php/cje-rce/article/view/2446 consulté le 15 février 2018.

Chatigny, C., Nadon-Vézina, L., Riel, J., Couture, V., \& Hastey, P. (2012). Analyse ergonomique de la santé et de la sécurité en centre de formation professionnelle. Rapport IRSST. http://www.irsst.qc.ca/ media/documents/PubIRSST/R-756.pdf?v=2018-07-02

Clénet, J. (1998). Représentations, formation et alternance. Paris : L'Harmattan.

Conseil supérieur de l'éducation (2004). Un nouveau souffle pour la profession enseignante. SainteFoy CSE.

Coulombe, S., Zourhlal, A., \& Allaire, S. (2010). Des obstacles à l'insertion des nouveaux enseignants en formation professionnelle. Formation et profession, 17(2), 25-28.

Cros. F., \& Raisky, C. (2010). Autour des mots de la formation. « Référentiel ». Recherche \& formation, 64, 105-116.

Davezies, P. (2012). Enjeux, difficultés et modalités de l'expression sur le travail : point de vue de la clinique médicale du travail. Perspectives interdisciplinaires sur le travail et la santé [en ligne], 14(2), http://journals.openedition.org/pistes/2566

Deschenaux, F., \& Roussel, C. (2008). L'accès à la carrière enseignante en formation professionnelle au secondaire : le choix d'un espace professionnel. Nouveaux cahiers de la recherche en éducation, 11(1), 1-16. 
Deschenaux, F., \& Roussel, C. (2010). De la pratique à l'enseignement d'un métier : l'obligation de formation universitaire comme événement marquant du parcours professionnel. Éducation et francophonie, XXXVIII, 92-108.

Deschenaux, F., \& Roussel, C. (2011). L'expérience de métier : le catalyseur des conceptions de l'enseignement en formation professionnelle au Québec. Recherches en éducation, 11, 15-26.

Deschenaux, F., \& Tardif, M. (2016a). Une enquête sur la formation à l'enseignement professionnel. Faits saillants et enjeux. In C. Gagnon \& S. Coulombe (Eds), Enjeux et défis de la formation à l'enseignement professionnel (p. 233-262). Québec : Presses de l'Université du Québec. Deschenaux, F., \& Tardif, M. (2016b). Devenir enseignant en formation professionnelle au Québec : la collaboration entre les acteurs favorise-t-elle la persévérance ? Revue canadienne de l'éducation, 39(1), 1-23. http://journals.sfu.ca/cje/index.php/cje-rce/article/view/2093/1837

Diarra, M. (2011). Formation des formateurs : place dans une éducation de qualité des adultes. International Review of Education, 57(1/2), 145-159.

Ducrey, F., Hrizi, Y., \& Issaieva Moubarak-Nahra, E. (2011). Analyse de la charge de travail des enseignants des centres de formation professionnelle. Note d'information du SRED. https:// www.ge.ch/recherche-education/doc/publications/notesinfo/notes-sred-46.pdf

Fournier, G., Bourassa, B., \& Gauthier, C. (2013). Introduction. In G. Fournier, B. Bourassa, \& C. Gauthier (Eds.), Les dynamiques d'insertion et d'intégration en emploi dans tous leurs états : événementsclés et enjeux particuliers du cheminement professionnel (p. 1-14). Québec : Les Presses de l'Université Laval.

Gagnon, C. (2008). Arrimage des pratiques éducatives d'enseignants et de formateurs en entreprise en contexte d'alternance. Recherches qualitatives, 27(1), 141-190.

Gagnon, C. (2013). Analyse de l'échec du stage en enseignement professionnel : perceptions de formateurs quant aux difficultés des stagiaires et à l'accompagnement fourni. Dans J.-F. Desbiens, C. Spallanzani, \& C. Borges (Eds.), Quand le stage en enseignement déraille. Regards pluriels sur une réalité trop souvent occultée (p. 117-146). Québec : Presses de l'Université du Québec.

Gagnon, C., \& Coulombe, C. (2016). Enjeux et défis de la formation à l'enseignement professionnel. Québec : Presses de l'Université du Québec.

Grossmann, S. (2011). Des enseignants qui res(is)tent : dynamiques identitaires et investissement du champ de l'enseignement professionnel. Recherches en éducation, 11, 67-82.

Habboub, E., Lenoir, Y., \& Tardif, M. (2008). La didactique professionnelle et la didactique des savoirs professionnels dans la documentation scientifique : un essai de synthèse des travaux francophones. In Y. Lenoir \& P. Pastré (Eds), Didactique professionnelle et didactiques disciplinaires en débat (p. 21-52). Toulouse : Octarès.

Holgado, O., \& Nizet, I. (2016). Évaluation et dilemmes d'enseignants au Québec. Éducation Permanente, hors-série AFPA 2016, 123-132.

Jellab, A. (2005). Le travail enseignant en lycée professionnel et ses paradoxes. Sociologie du Travail, 47(4), 502-517.

Ledent, C., \& Schevers, P. (2016). Comment les professeurs de pratique professionnelle développent-ils leurs identités dans leurs relations aux individus et au contexte scolaire? Une identité entre deux mondes? Mémoire de recherche, École d'éducation et de formation, Louvain-La-Neuve, Belgique.

Leplat, J. (1992). L'analyse du travail en psychologie ergonomique. Toulouse : Octarès. 
Leplat, J. (1997). Regards sur l'activité en situation de travail. Contribution à la psychologie ergonomique. Paris : PUF.

Leplat, J. (2000). L'analyse psychologique de l'activité en ergonomie. Toulouse : Octarès.

Leplat, J. (2008). Repères pour l'analyse de l'activité en ergonomie. Paris : PUF.

Leplat, J., \& Hoc, J.M. (1983). Tâche et activité dans l'analyse psychologique des situations. Cahiers de psychologie cognitive, 3(1), 49-63. Réédité dans Leplat J. (Ed.) L'analyse du travail en psychologie ergonomique. Toulouse : Octarès.

Masdonati, J., Fornier, G., \& Pinault, M. (2015). La formation professionnelle au Québec : le regard des élèves. L'orientation scolaire et professionnelle [en ligne], 44(2). URL : http:// journals.openedition.org/osp/4590

Mayen, P. (2008). Dix développements sur la didactique professionnelle et le développement. Dans Y. Lenoir, \& P. Pastré (Eds.), Didactique des savoirs professionnels, didactique professionnelle (p. 110-125). Toulouse : Octarès.

Mayen, P. (2012). Les situations professionnelles : un point de vue de didactique professionnelle. Phronesis, 1(1), 59-67.

MELS (2005). Cadre de référence sur la planification des activités d'apprentissage et d'évaluation. Québec : Gouvernement du Québec. Ministère de l'Éducation, du Loisir et du Sport. http:// www.inforoutefpt.org/ministere_docs/AdminInfo/CadreRef/CadreReference.pdf

MELS (2014). Indicateurs de l'éducation. Québec : Gouvernement du Québec, Ministère de l'Éducation, du Loisir et du Sport. http://www.education.gouv.qc.ca/fileadmin/site_web/ documents/PSG/statistiques_info_decisionnelle/indicateurs_2014_fr.pdf

MEQ (2001). La formation à l'enseignement. Les orientations, les compétences professionnelles. Québec : Gouvernement du Québec, Ministère de l'Éducation. http://www.education.gouv.qc.ca/ fileadmin/site_web/documents/reseau/formation_titularisation/ formation_enseignement_orientations_EN.pdf

Ochanine, D.A. (1969). Rôle de l'image opérative dans la saisie du contenu informationnel des signaux. Questions de Psychologie, 4. Repris in L'image opérative. Actes de séminaire (1-5 juin 1981) et recueil d'articles D. Ochanine. Paris : Université de Paris I.

Ochanine, D.A., \& Koslov, V. (1971). L'image opérative effectrice. Question de Psychologie, 3. Repris in L'image opérative. Actes d'un séminaire (1-5 juin 1981) et recueil d'articles D. Ochanine. Paris : Université de Paris I.

Ochanine, D.A., \& Zaltzman, A. (1973). Opérativité de l'image d'un processus de contrôle. In Régulation de l'activité. Moscou. Repris in L'image opérative. Actes d'un séminaire (1-5 juin 1981) et recueil d'articles D. Ochanine. Paris : Université de Paris I.

Payeur, C. (1994). Les récentes politiques de formation professionnelle au Québec : vers un nouveau rapport éducatif? Critique régionale, 23-24, 1-18.

Pastré, P. (2011). La didactique professionnelle. Approche anthropologique du développement chez les adultes. Paris : Presses Universitaires de France.

Rogalski, J. (2013). Les représentations en psychologie du travail, de la formation et en didactique des disciplines scientifiques. Recherches en éducation, 17, 73-89.

Roussel, C. (2014). Analyse du niveau de complexité de situations évaluatives de compétences utilisées par des enseignantes et des enseignants de la formation professionnelle au secondaire : le cas du programme de Santé, assistance et soins infirmiers. Thèse de doctorat, Université de Sherbrooke. 
Santelmann, P. (2014). Transformation du travail et pédagogie en formation des adultes. Éducation permanente, Hors-série AFPA 2014, 9-19.

Saussez, F., \& Yvon, F. (2014). Problématiser l'usage de la co-analyse de l'activité en formation initiale à l'enseignement. In L. Paquay, P. Perrenoud, M. Altet, J. Desjardins, \& R. Etienne (Eds.), Travail réel des enseignants et formation. Quelle référence au travail des enseignants dans les objectifs, les dispositifs et les pratiques? (p. 113-126). Bruxelles : De Boeck.

Tardif, M. (2012). Les enseignants au Canada : une vaste profession sous pression. Formation et profession, 20(1), 1-8. http://dx.doi.org/10.18162/fp.2012.172

Tardif, M., \& Deschenaux, F. (2014). L'abandon des études universitaires en formation à l'enseignement professionnel : un phénomène difficile à cerner. Phronesis, 3, 78-89.

Teiger, C. (1993). Représentations du travail, travail de la Représentation. In A. Weill- Fassina, A., P. Rabardel, \& D. Dubois (Eds). Représentations pour l'action (p 311-344). Toulouse : Octarès.

Valot, C., Grau, J.C., \& Amalberti, A. (1993). Les métaconnaissances : des représentations de ses propres connaissances. In A. Weill-Fassina, P. Rabardel, \& D. Dubois (Eds), Représentations pour l'action (p 271-293). Toulouse : Octarès.

Vergnaud, G. (2007). Réponse de Gérard Vergnaud. In M. Merri (Ed.), Activité humaine et conceptualisation: Questions à Gérard Vergnaud (p. 341-357). Toulouse : Presses Universitaires du Mirail.

Vermersch, P., \& Weill-Fassina, A. (1981). Image opérative ou représentation fonctionnelle ? 1. À Propos de quelques difficultés sémantiques. 2. Intérêt pour la conception et l'utilisation d'intermédiaires graphiques. Dans L'image opérative, Actes d'un séminaire et recueil d'articles d'Ochanine, Université de Paris 1, p. 44-82.

Weill-Fassina, A., Rabardel, P., \& Dubois, D. (1993). Représentations pour l'action. Toulouse : Octarès.

\section{NOTES}

1. Dans ce texte, le terme «charge de travail» ne réfère pas au concept tel qu'utilisé en ergonomie, mais est employé dans son sens commun, soit celui de la somme des tâches professionnelles et des responsabilités attribuées à une personne dans le cadre de sa mission ou de sa fonction professionnelle (dictionnaire Larousse en ligne).

\section{RÉSUMÉS}

Cet article rend compte d'une recherche qui explore le travail quotidien de huit enseignants de la formation professionnelle secondaire au Québec, dans six métiers : arpentage, cuisine, entretien paysager, secrétariat, transport par camion et conduite des engins de chantier. Menée selon une démarche d'analyse du travail à partir d'entretiens libres, la recherche se propose d'accéder aux représentations des enseignants participants afin de rendre compte de la manière dont ceux-ci se représentent leur propre métier, les tâches qui le composent, les responsabilités qui leur incombent, ainsi que les conditions de travail, les difficultés qu'ils rencontrent et les moyens 
qu'ils déploient pour y faire face au quotidien. Les résultats obtenus montrent le partage entre les tâches prescrites et celles qui sont prises en charge volontairement par les enseignants, dans le but de favoriser l'apprentissage des élèves et d'assurer le bon fonctionnement de leur centre de formation. Nous constatons que cela amène une surcharge de travail dont la réalisation se prolonge sur le temps personnel, conduisant à une délimitation floue entre vie professionnelle et vie privée.

This paper attempts to provide an exploratory overview of the daily work of eight teachers from secondary vocational education in Quebec. Six skilled trades are involved: surveying, cooking, landscape maintenance, secretarial work, trucking and driving construction machinery. The study was carried out using a work analysis approach based on open-ended interviews. It aimed to access the representations of participating teachers in order to give an account of how they see their own profession, the tasks of which it is composed and the responsibilities that are theirs, as along with the working conditions, the difficulties they encounter and the means they deploy to deal with the latter on a daily basis. The results show the sharing between prescribed tasks and those voluntarily undertaken by teachers, with the aim of promoting student learning and ensuring the proper functioning of their training centre. We note that this leads to a work overload, the completion of which extends into personal time, leading to a blurred line between professional and private life.

\section{INDEX}

Mots-clés : travail enseignant, formation professionnelle, didactique professionnelle, analyse du travail

Keywords : teacher's work, vocational training, professional didactics, work analysis

\section{AUTEUR \\ OTILIA HOLGADO}

CREAS, Université de Sherbrooke - Otilia.holgado@usherbrooke.ca 